\title{
Current Status and Influencing Factors of the Quality of Work Life of Nurses in Intensive Care Unit
}

\author{
Hong Hu, Hong Zhou*, Jing Geng, Liu Zhang \\ School of Medicine, Yangtze University, Jingzhou, China \\ Email: ^1059634547@qq.com
}

How to cite this paper: $\mathrm{Hu}, \mathrm{H}$., Zhou, H., Geng, J. and Zhang, L. (2020) Current Status and Influencing Factors of the Quality of Work Life of Nurses in Intensive Care Unit. Yangtze Medicine, 4, 183-192. https://doi.org/10.4236/ym.2020.43018

Received: November 28, 2019

Accepted: June 27, 2020

Published: June 30, 2020

Copyright (อ 2020 by author(s) and Scientific Research Publishing Inc. This work is licensed under the Creative Commons Attribution International License (CC BY 4.0).

http://creativecommons.org/licenses/by/4.0/ (c) (i) Open Access

\begin{abstract}
Objective: To investigate the current status and influencing factors of the quality of work life of nurses in Intensive Care Unit (ICU). Methods: A total of 243 ICU nurses from 6 general hospitals in Jingzhou were selected by convenient sampling method. Questionnaires were conducted with the general information questionnaire, Connor-Davidson Resilience Scale (CD-RISC) and Work-Related Quality of Life Scale-2 (WRQoL-2). Results: The total average score of the quality of work life of ICU nurses was (3.35 \pm 0.44$)$, which was at a medium level. Department, professional title, frequency of night shift, experience of workplace violence and psychological resilience were the influencing factors of the quality of work life of nurses in ICU, accounting for $39.4 \%$ of the variance. Conclusion: The quality of work life of ICU nurses needs to be improved. Nursing managers should pay attention to the work environment of ICU nurses, rationally allocate manpower, and improve the status of psychological resilience of nurses, so as to improve their quality of work life.
\end{abstract}

\section{Keywords}

ICU, Nurse, Quality of Work Life, Psychological Resilience, Influencing Factors

\section{Introduction}

The National Nursing Career Development Plan (2016-2020) pointed out that one of the main tasks of the development of nursing industry during the 13th Five-Year Plan period was to improve the quality of nursing services and pro- 
mote high-quality nursing [1]. The quality of work life of nurses is directly proportional to the quality of care services. Having a good quality of work and life is a prerequisite for nurses to carry out high-quality nursing and contribute to the sustainable development of health service systems [2]. The Intensive Care Unit (ICU) is a department that treats all kinds of severe illness and multi-systemic failure patients. Its nursing work has the characteristics of high risk, high tension and high labor intensity. ICU nurses have higher work pressure, long-term stress, and poor quality of work life [3] [4] [5]. At present, domestic research on the quality of nursing work life focuses on the emergency department and pediatrics department. There are few studies on the quality of work life of ICU nurses, and it is limited to the top three hospitals. It is extremely urgent to carry out research on the quality of work life of ICU nurses. Therefore, this study aims to investigate the current status and influencing factors of the quality of work life of ICU nurses in different grades of hospitals, and provide reference for the intervention study to improve the quality of work life of ICU nurses.

\section{Object and Method}

\subsection{Research Object}

From January to April of 2019, the ICU nurses in 6 general hospitals (3 tertiary general hospitals and 3 secondary general hospitals) in Jingzhou were selected as research objects by convenient sampling method. Inclusion criteria: work in clinical care in ICU; work experience in ICU $\geq 1$ year; volunteer to participate in this survey. Exclusion criteria: those who are unable to complete the questionnaire for various reasons; non-unit nurses, such as training nurses.

\subsection{Research Tools}

\subsubsection{General Information Questionnaire}

The general information questionnaire was designed by consulting domestic and foreign related literatures [2] [6] [7], and reviewed by 5 nursing management experts, and revised with expert opinions. Including gender, age, hospital grade, department, working years, working years in ICU, marital status, education level, professional title, position, frequency of night shift, monthly income, and experience of workplace violence.

\subsubsection{Work-Related Quality of Life Scale}

Based on Van Laar et al. [8], Shao Ya et al. [9] translated and revised the Work-Related Quality of Life Scale-2 (WRQoL-2), including 7 factors, 33 items. Using the Likert 5 rating ( $1=$ very disagree, $2=$ disagree, $3=$ no opinion, $4=$ agree, $5=$ very agree), convert the score of the reverse items and then calculate the score. The scores of the scale range from 33 to 165 . The higher the score of the scale, the higher the quality of work life. The content validity CVI of the scale is 0.91 ; the total Cronbach's $\alpha$ coefficient is 0.939 , and the factors are between 0.652 and 0.859 . 


\subsubsection{Psychological Resilience Scale}

Based on Connor et al. [10], Li Yameng et al. [11] translated and revised the Connor-Davidson Resilience Scale (CD-RISC), including 3 factors (adaptability, toughness, goal achievement), 23 items. Using the Likert 5 rating $(0=$ never, $1=$ rarely, 2 = sometimes, 3 = often, $4=$ always), the scores of the scale range from 0 to 92 .The higher the score, the better the psychological resilience. The total Cronbach's $\alpha$ coefficient of the scale is 0.940 , and the factors are between 0.803 and 0.903 .

\subsection{Survey Method}

This study was approved by the hospital ethics committee. The questionnaire of this study included 7 factors of WRQoL-2 and 3 factors of CD-RISC. The estimation formula of the sample size was [Max (dimension number)*(15-20) $]^{*}[1$ $+(15 \%-20 \%)]$. It was calculated that the sample size of this study needed 173 240. Under the coordination of the nursing department and the head nurses of each hospital, the nursing staff would be concentrated in the department. After obtaining the consent of the respondents, the anonymous survey was adopted. The purpose, significance and filling method of the investigation would be explained to the respondents before the investigation. After the questionnaire was filled out, checked it on the spot. A total of 260 questionnaires were distributed in this study. After eliminating the response absence rate of $>10 \%$ or selecting the same option, 243 valid questionnaires were collected, with an effective rate of $93.5 \%$.

\subsection{Statistical Method}

SPSS22.0 software was used for statistical analysis. The general data and the scores of each scale were described by Mean \pm SD, frequency, and percentage. The $t$-test or $F$-test was used to compare the scores of the quality of work life of ICU nurses with different demographic characteristics. The analysis of the factors influencing the quality of work life of ICU nurses was performed by multiple linear regression analysis. The difference was statistically significant $(P<$ 0.05).

\section{Results}

\subsection{The Scores of the Quality of Work Life and Psychological Resilience of ICU Nurses}

The total score of the quality of work life of 243 ICU nurses was (110.60 \pm $14.61)$, and the total average score was $(3.35 \pm 0.44)$. The factors divided into low to high were work pressure $(2.82 \pm 0.51)$, work conditions $(3.25 \pm 0.62)$, general well-being $(3.34 \pm 0.59)$, work evaluation $(3.47 \pm 0.56)$, work-family balance ( $3.53 \pm 0.75)$, work control $(3.59 \pm 0.52)$, career satisfaction $(3.64 \pm 0.50)$. The score of psychological resilience was $(55.83 \pm 12.23)$, and the total average score was $(2.43 \pm 0.53)$. The score of adaptability dimension was $(2.21 \pm 0.49)$, followed by toughness $(2.51 \pm 0.60)$ and goal achievement $(2.71 \pm 0.62)$. 


\subsection{Comparison of the Scores of the Quality of Work Life of ICU Nurses with Different Demographic Characteristics}

There were significant differences in scores of the quality of work life of ICU nurses in different departments, titles, professional positions, frequencies of night shift, and experiences of workplace violence $(P<0.05)$. These were shown in Table 1.

\subsection{Multiple Linear Regression Analysis on the Influencing Factors of the Quality of Work Life of ICU Nurses}

Taking the total average score of the quality of work life of ICU nurses as the dependent variable, and the department, professional title, position, frequency of night shift, experience of workplace violence and the total score of psychological resilience as the independent variable, the multiple linear regression analysis was carried out. The assignment of the independent variable was shown in Table 2. The results of multiple regression analysis showed that department, professional title, frequency of night shift, experience of workplace violence and psychological resilience were the influencing factors of the quality of work life of ICU nurses, accounting for $39.4 \%$ of the variance. The results were shown in Table 3 .

\section{Discussion}

\subsection{The Importance of Investigating the Quality of Work Life of ICU Nurses}

Lee et al. found that as many as 720 nurses $(56.1 \%)$ had a turnover intention through a survey of 1283 hospital nurses in Taiwan [12]. The score of work stressor of ICU nurses was higher than that of other department nurses [4], and the turnover intention of ICU nurses was higher than that of general outpatient nurses [13]. As the core department of the hospital, the stability of ICU nursing team is the key point that hospital managers need to pay attention to [14]. Studies have shown that the lower the quality of work life of nurses, the stronger their tendency to leave [15]. Through the investigation of the status quo of the quality of work life of ICU nurses, the hospital nursing management system can be prospectively reviewed, the weak links can be found, and nursing managers can take corresponding measures to improve the quality of work life of ICU nurses and prevent ICU nurses from losing. At the same time, the improvement of the quality of work life of nurses will improve the well-being and satisfaction of nurses, leading to beneficial outcomes for hospitals and patients, such as higher patient satisfaction [16] [17].

\subsection{The Quality of Work Life of ICU Nurses Needs to Be Improved}

In this study, the total average score of the quality of work life of ICU nurses was $(3.35 \pm 0.44)$, which was at a medium level, and the situation was not optimistic. Among them, the two factors of work pressure and work conditions had the lowest score, which may be related to the following reasons: 1) ICU was a 
Table 1. Comparison of the scores of the quality of work life of ICU nurses with different demographic characteristics $(n=243)$.

\begin{tabular}{|c|c|c|c|c|}
\hline Characteristics & Cases (\%) & Average score $(X \pm S)$ & $t / F$-value & $P$-value \\
\hline Gender & & & $-0.678^{\#}$ & 0.502 \\
\hline Meal & $28(11.5)$ & $3.29 \pm 0.49$ & & \\
\hline Female & $215(88.5)$ & $3.36 \pm 0.44$ & & \\
\hline Age & & & 2.191 & 0.071 \\
\hline 21 - 25 years old & $53(21.8)$ & $3.47 \pm 0.36$ & & \\
\hline 26 - 30 years old & $101(41.6)$ & $3.31 \pm 0.46$ & & \\
\hline 31 - 35 years old & $50(20.6)$ & $3.24 \pm 0.46$ & & \\
\hline 36 - 40 years old & $22(9.1)$ & $3.43 \pm 0.35$ & & \\
\hline$\geq 41$ years old & $17(7.0)$ & $3.45 \pm 0.56$ & & \\
\hline Hospital grade & & & $-0.692^{\#}$ & 0.491 \\
\hline Tertiary hospital & $192(79.0)$ & $3.28 \pm 0.43$ & & \\
\hline Second-class hospital & $51(21.0)$ & $3.32 \pm 0.39$ & & \\
\hline Department & & & $-2.371^{\#}$ & $0.019^{*}$ \\
\hline Comprehensive ICU & $105(43.2)$ & $3.28 \pm 0.43$ & & \\
\hline Specialist ICU & $138(56.8)$ & $3.41 \pm 0.44$ & & \\
\hline Working years & & & 2.092 & 0.082 \\
\hline $1-5$ years & $102(42.0)$ & $3.40 \pm 0.42$ & & \\
\hline $6-10$ years & $79(32.5)$ & $3.25 \pm 0.47$ & & \\
\hline $11-15$ years & $41(16.9)$ & $3.35 \pm 0.39$ & & \\
\hline $16-20$ years & $7(2.9)$ & $3.63 \pm 0.60$ & & \\
\hline$\geq 21$ years & $14(5.8)$ & $3.39 \pm 0.46$ & & \\
\hline Working years in ICU & & & 1.336 & 0.257 \\
\hline $1-5$ years & $119(49.0)$ & $3.40 \pm 0.41$ & & \\
\hline $6-10$ years & $84(34.6)$ & $3.27 \pm 0.49$ & & \\
\hline $11-15$ years & $28(11.5)$ & $3.35 \pm 0.39$ & & \\
\hline 16 - 20 years & $5(2.1)$ & $3.44 \pm 0.34$ & & \\
\hline$\geq 21$ years & $7(2.9)$ & $3.44 \pm 0.64$ & & \\
\hline Marital status & & & $1.571^{\#}$ & 0.118 \\
\hline Unmarried & $87(35.8)$ & $3.41 \pm 0.46$ & & \\
\hline Married & $156(64.2)$ & $3.32 \pm 0.43$ & & \\
\hline Education level & & & 0.351 & 0.704 \\
\hline Associate degree & $35(14.4)$ & $3.41 \pm 0.38$ & & \\
\hline Bachelor & $204(84.0)$ & $3.34 \pm 0.45$ & & \\
\hline Postgraduate & $4(1.6)$ & $3.28 \pm 0.54$ & & \\
\hline Professional title & & & 2.924 & $0.035^{\star}$ \\
\hline Nurse & $76(31.3)$ & $3.44 \pm 0.40$ & & \\
\hline Nurse practitioner & $96(39.5)$ & $3.34 \pm 0.42$ & & \\
\hline Supervisor & $64(26.3)$ & $3.28 \pm 0.51$ & & \\
\hline Deputy chief nurse and above & $7(2.9)$ & $3.63 \pm 0.33$ & & \\
\hline
\end{tabular}




\section{Continued}

\begin{tabular}{ccccc}
\hline Position & & & 4.677 & $0.010^{*}$ \\
Nurse & $186(76.5)$ & $3.36 \pm 0.44$ & & \\
Nursing/Teaching team leader & $44(18.1)$ & $3.24 \pm 0.44$ & & \\
Head nurse & $13(5.3)$ & $3.66 \pm 0.39$ & & \\
Frequency of night shift & & & 3.745 & $0.012^{*}$ \\
None & $40(16.5)$ & $3.45 \pm 0.36$ & & \\
$1-4$ times/month & $58(23.9)$ & $3.43 \pm 0.47$ & & \\
$5-8$ times/month & $83(34.2)$ & $3.35 \pm 0.44$ & & \\
Over 8 times/month & $62(25.5)$ & $3.21 \pm 0.44$ & & \\
Monthly income & & & & \\
$<3000$ yuan & $9(3.7)$ & $3.31 \pm 0.29$ & & \\
3000 - 4999 yuan & $67(27.6)$ & $3.43 \pm 0.35$ & & \\
5000 - 6999 yuan & $116(47.7)$ & $3.33 \pm 0.44$ & & \\
7000 - 8999 yuan & $43(17.7)$ & $3.30 \pm 0.58$ & & \\
$>9000$ yuan & $8(3.3)$ & $3.34 \pm 0.54$ & & \\
Yes & & & & \\
No & $107(44.0)$ & $3.23 \pm 0.41$ & & \\
Experience of workplace violence & $136(56.0)$ & $3.45 \pm 0.45$ & & \\
\hline
\end{tabular}

${ }^{*}: P<0.05 ;{ }^{* *}: P<0.01$; * $t$-value.

Table 2. Assignment of the independent variable.

\begin{tabular}{|c|c|}
\hline Variable & Assignment \\
\hline Department & Comprehensive ICU = 1; Specialist ICU $=2$ \\
\hline Professional title & $\begin{array}{l}\text { Nurse }=1 ; \text { Nurse practitioner }=2 \text {; Supervisor }=3 \text {; } \\
\text { Deputy chief nurse and above }=4\end{array}$ \\
\hline Position & $\begin{array}{l}\text { Nurse }=1 ; \text { Nursing/Teaching team leader }=2 ; \\
\text { Head nurse }=3\end{array}$ \\
\hline Frequency of night shift & $\begin{array}{l}\text { None }=1 ; 1-4 \text { times } / \text { month }=2 ; \\
5-8 \text { times } / \text { month }=3 \text {; over } 8 \text { times } / \text { month }=4\end{array}$ \\
\hline Experience of workplace violence & No $=1 ;$ Yes $=2$ \\
\hline Score of psychological resilience & Substitution of original value \\
\hline
\end{tabular}

Table 3. Multiple linear regression analysis of factors influencing the quality of work life of ICU nurses $(\mathrm{n}=243)$.

\begin{tabular}{cccccc}
\hline Variable & $B$ & $S E$ & $\beta$ & $t$ & $P$ \\
\hline Constant term & 2.105 & 0.185 & & 11.370 & $<0.001$ \\
Score of psychological resilience & 0.020 & 0.002 & 0.547 & 10.724 & $<0.001$ \\
Experience of workplace violence & 0.146 & 0.046 & 0.164 & 3.145 & 0.002 \\
Department & 0.118 & 0.047 & 0.132 & 2.531 & 0.012 \\
Professional title & -0.067 & 0.028 & -0.126 & -2.401 & 0.017 \\
Frequency of night shift & -0.052 & 0.023 & -0.120 & -2.243 & 0.026 \\
\hline
\end{tabular}

$R^{2}=0.394, F=32.490, P<0.001$. 
department for treating critical patients, most of patients can't take care of themselves, so nurses had a large amount of nursing work. 2) Heavy workload, sudden emergencies, frequent night shifts, easy to lead to lower physical fitness and energy loss; 3) The special nature of ICU nursing work required the nurses to have a higher level of knowledge and skills, and to be proficient in the use and maintenance of various types of monitoring equipment. 4) The working environment of the ICU was closed and noisy, and various monitoring instruments had frequent alarms, which may lead to anxiety and fatigue of the nurses. At the same time, the ICU nurses in this study generally had lower scores of general well-being, work evaluation, and work-family balance, which may be related to the following reasons: 1) $62.2 \%$ of respondents were 26 - 35 years old, except the heavy workload of ICU nursing, they had be responsible for teaching, scientific research, etc., easily led to imbalance between work and family life. 2) ICU nurses with a working experience of less than 10 years accounted for $74.5 \%$, showing obvious rejuvenation, lack of clinical experience, unable to effectively deal with emergencies, and reduced work evaluation. 3) $44 \%$ of ICU nurses in this study experienced workplace violence, and there were concerns about work environment, career development and their own safety, resulting in a decrease in general well-being.

\subsection{Factors Influencing the Quality of Work Life of ICU Nurses}

\subsubsection{The Influence of Demographic Characteristics on the Quality of Work Life of ICU Nurses}

The results of this study showed that department, professional title, frequency of night shift, and experience of workplace violence were the influencing factors of the quality of worklife of ICU nurses, and the scores were statistically significant $(P<0.05)$. The quality of work life of nurses in specialist ICU was higher than that of comprehensive ICU nurses. It may be due to the relatively single disease of specialist ICU patients, and most of them were postoperative patients, the condition was relatively stable, and the nursing workload was relatively lighter [18]. The ICU nurses with deputy chief nurses and above titles had higher scores of the quality of work life, and the scores of the quality of work life of head nurses were higher than those of ordinary nurses. It may be due to the rich clinical experience of this group of people, which can arrange working hours reasonably, and they were mainly engaged in nursing management and scientific research and had lower work pressure. ICU nurses with frequent night shifts had lower scores of the quality of work life. It may be due to frequent night shifts, resulting in irregular sleep, reduced the quality of sleep, prone to anxiety and depression, and nurses may face emergencies such as rescue at any time, the state of mental stress was high and the body load was too heavy [19] [20]. ICU nurses who had experienced workplace violence had lower scores of the quality of work life, which may be due to the fact that workplace violence had a certain degree of impact on their physical and mental health, and work pressure was increasing. It is suggested that nursing managers should pay attention to the con- 
struction of ICU work environment, create a positive and healthy work atmosphere for nurses, and increase the intensity of introducing and retaining talents. At the same time, according to the age and ability of nurses, the labor and frequency of night shift can be reasonably allocated to reduce the workload of nurses.

\subsubsection{The Influence of Psychological Resilience on the Quality of Work Life of ICU Nurses}

The results of this study showed that the score of ICU nurses' psychological resilience was $(55.83 \pm 12.23)$, which was at a medium level. The results of regression analysis showed that psychological resilience was the main influencing factor of the quality of work life. Mainly manifested in: 1) Psychological resilience emphasized the ability of individuals to effectively cope and actively adapt to adverse conditions [14], high professional expectations and fast-paced work environment would make ICU nurses face the risk of high-intensity fatigue and stress-related diseases [21], low psychological resilience may lead to maladaptation, showing mental illness such as anxiety and depression [22]; it was likely to lead to the occurrence of an error event and a reduction in job evaluation; 2) ICU nurses were repeatedly faced with the end of life, prolonging life through artificial support measures, providing end-of-life care, etc., due to lack of reaction time and experience in dealing with similar incidents, it would cause tension, panic and psychological burden. However, psychological resilience can improve the individual's ability to face and adapt to trauma, tragedy, threats or major stressors [23]; 3) The results of Hudgins et al. [24] showed that psychological resilience played a vital role in improving nurses' work satisfaction. Low level of psychological resilience can lead to negative factors in nurses' negative coping work. Therefore, nursing managers should strengthen the intervention of ICU nurses' psychological resilience, actively carry out psychological counseling for nurses, and provide psychological assistance when necessary. At the same time, outdoor group activities can be properly carried out to foster a harmonious organizational atmosphere, to create a place for ICU nurses to vent their emotions and stress, and to alleviate work pressure.

\section{Conclusion}

In summary, the quality of work life and psychological resilience of ICU nurses were at a medium level, and the situation was not optimistic. Department, professional title, frequency of night shift, experience of workplace violence and psychological resilience were the influencing factors of the quality of work life of ICU nurses. Nursing managers should allocate manpower rationally and take appropriate measures to improve the psychological resilience of ICU nurses, thus improving their quality of work life. In this study, only 243 ICU nurses from 6 general hospitals in Jingzhou were investigated, and the number of samples was limited to some extent, and the results only represented the status quo of ICU nurses within the scope of investigation. After that, it plans to carry out 
large sample survey, and carry out intervention research on the quality of work life of ICU nurses, so as to formulate feasible measures for improving the quality of work life of ICU nurses, and lay a good foundation for promoting the quality of ICU nursing service and promoting high-quality nursing.

\section{Funding Projects}

Scientific Research Fund of Jingzhou Science and Technology Bureau (2019EC61-18).

\section{Conflicts of Interest}

The authors declare no conflicts of interest regarding the publication of this paper.

\section{References}

[1] National Health and Family Planning Commission (2016) National Nursing Career Development Plan (2016-2020).

[2] Nowrouzi, B., Giddens, E., Gohar, B., et al. (2016) The Quality of Work Life of Registered Nurses in Canada and the United States: A Comprehensive Literature Review. International Journal of Occupational and Environmental Health, 22, 341-358. https://doi.org/10.1080/10773525.2016.1241920

[3] Deng, W. and Li, X.Z. (2019) Research Progress on Sympathetic Fatigue of ICU Nurses. Chinese Nursing Research, 33, 459-463.

[4] Liu, H., Jing, X.B., Fang, X.J., et al. (2016) Pathway Analysis of Job Stressors and Psychological Capital on Career Development of ICU Specialist Nurses. Chinese Journal of Nursing, 51, 1012-1016.

[5] Chen, B.Q. and Wang, B.C. (2014) Survey of Quality of Work Life in ICU Nurses in Class III Grade I Hospital. Nursing Journal of Chinese People's Liberation Army, 31, 17-19, 23.

[6] Akter, N., Akkadechanunt, T., Chontawan, R., et al. (2018) Factors Predicting Quality of Work Life among Nurses in Tertiary-Level Hospitals, Bangladesh. International Nursing Review, 65, 182-189. https://doi.org/10.1111/inr.12401

[7] Yin, X.G., Zhou, Y.F., Gu, Z.J., et al. (2017) Current Status and Influencing Factors of Quality of Working Life among Nurses in Emergency Department. Chinese Nursing Management, 17, 659-663.

[8] Van, Laar, D., Edwards, J.A. and Easton, S. (2007) The Work-Related Quality of Life Scale for Healthcare Workers. Journal of Advanced Nursing, 60, 325-333. https://doi.org/10.1111/j.1365-2648.2007.04409.x

[9] Shao, Y., Liao, S.L., Zhong, H.Q., et al. (2014) Work-Related Quality of Life Scale among Chinese Nurses: Evaluation of the Reliability and Validity. Journal of Nursing Science, 29, 1-3.

[10] Connor, K.M. and Davidson, J.R. (2003) Development of a New Resilience Scale: The Connor-Davidson Resilience Scale (CD-RISC). Depress Anxiety, 18, 76-82. https://doi.org/10.1002/da.10113

[11] Li, Y.M., Wang, P., Shan, Y., et al. (2014) Reliability and Validity of the Connor-Davidson Resilience Scale in Nurses. Chinese Journal of Practical Nursing, 30, $5-8$

[12] Lee, Y.W., Dai, Y.T., Chang, M.Y., et al. (2017) Quality of Work Life, Nurses' Inten- 
tion to Leave the Profession, and Nurses Leaving the Profession: A One-Year Prospective Survey. Journal of Nursing Scholarship, 49, 438-444.

https://doi.org/10.1111/jnu.12301

[13] Feng, X.L., Shang, S.M., Liu, C.Y., et al. (2018) Job Characteristics, Job Satisfaction and Turnover Intention among ICU Nurses. Journal of Nursing Science, 33, 66-68.

[14] Mealer, M., Jones, J. and Meek, P. (2017) Factors Affecting Resilience and Development of Posttraumatic Stress Disorder in Critical Care Nurses. American Journal of Critical Care, 26, 184-192. https://doi.org/10.4037/ajcc2017798

[15] Lee, Y.W., Dai, Y.T. and McCreary, L.L. (2015) Quality of Work Life as a Predictor of Nurses' Intention to Leave Units, Organisations and the Profession. Journal of Nursing Management, 23, 521-531. https://doi.org/10.1111/jonm.12166

[16] Nowrouzi, B., Lightfoot, N., Carter, L., et al. (2015) The Relationship between Quality of Work Life and Location of Cross-Training among Obstetric Nurses in Urban Northeastern Ontario, Canada: A Population-Based Cross Sectional Study. International Journal of Occupational Medicine and Environmental Health, 28, 571-586. https://doi.org/10.13075/ijomeh.1896.00443

[17] Abbasi, M., Zakerian, A., Mehri, A., et al. (2015) Investigation into Effects of Work-Related Quality of Life and Some Related Factors on Cognitive Failures among Nurses. International Journal of Occupational Safety and Ergonomics, 23, 386-392. https://doi.org/10.1080/10803548.2016.1216991

[18] Wang, J., Wang, J.L., Zhou, S., et al. (2017) The Status and Influencing Factors of Alarm Fatigue towards Medical Devices among ICU Nurses. Chinese Journal of Nursing, 52, 211-215.

[19] Fu, C.H., Wu, Y., Xin, R.M., et al. (2018) Psychological Resilience of ICU Nurses in Three Grade General Hospital and Its Influencing Factors. Chinese Journal of General Practice, 16, 663-666.

[20] Huang, S., Ye, T.H., Zhu, H.Y., et al. (2017) The Effect of Maintaining Duration of Sleep after Night Shifts of Pediatric ICU Nurses. Chinese Nursing Management, 17, 1066-1069.

[21] Brennan, E.J. (2017) Towards Resilience and Wellbeing in Nurses. British Journal of Nursing, 26, 43-47. https://doi.org/10.12968/bjon.2017.26.1.43

[22] Jiang, M.E. (2015) Research Progress of Nurses' Resilience. Chinese Nursing Management, 15, 1220-1222.

[23] Tseng, H.M., Shih, W.M., Shen, Y.C., et al. (2018) Work Stress, Resilience, and Professional Quality of Life among Nurses Caring for Mass Burn Casualty Patients After Formosa Color Dust Explosion. Journal of Burn Care \& Research, 39, 798-804. https://doi.org/10.1093/jbcr/irx053

[24] Hudgins, T.A. (2016) Resilience, Job Satisfaction and Anticipated Turnover in Nurse Leaders. Journal of Nursing Management, 24, E62-E69.

https://doi.org/10.1111/jonm.12289 